OPEN ACCESS

Edited by:

Dongming Hou,

Boston Scientific, United States

Reviewed by:

Thach Nguyen

Methodist Hospital, United States Ramesh Daggubati,

West Virginia University, United States

*Correspondence:

Yi-Heng $L i$

heng@mail.ncku.edu.tw Chun-Hung Su

such197408@gmail.com

tThese authors have contributed equally to this work

Specialty section:

This article was submitted to Coronary Artery Disease,

a section of the journal

Frontiers in Cardiovascular Medicine

Received: 08 September 2021 Accepted: 27 December 2021

Published: 24 February 2022

Citation:

Huang S-W, Chen P-W, Feng W-H, Hsieh I-C, Ho M-Y, Cheng C-W, Yeh $\mathrm{H}-\mathrm{I}$, Chen $\mathrm{C}-\mathrm{P}$, Huang $\mathrm{W}-\mathrm{C}$, Fang C-C, Lin H-W, Lin S-H, Tsai C-F,

Su C-H and Li Y-H (2022) Impact of the Dual Antiplatelet Therapy Score on Clinical Outcomes in Acute Coronary Syndrome Patients Receiving P2Y12 Inhibitor Monotherapy.

Front. Cardiovasc. Med. 8:772820. doi: 10.3389/fcrm.2021.772820

\section{Impact of the Dual Antiplatelet Therapy Score on Clinical Outcomes in Acute Coronary Syndrome Patients Receiving P2Y12 Inhibitor Monotherapy}

\author{
Sheng-Wei Huang ${ }^{1 \dagger}$, Po-Wei Chen ${ }^{2 \dagger}$, Wen-Han Feng ${ }^{3}$, I-Chang Hsieh ${ }^{4}$, Ming-Yun Ho ${ }^{4}$, \\ Chung-Wei Cheng ${ }^{5}$, Hung-I Yeh ${ }^{5}$, Ching-Pei Chen ${ }^{6}$, Wei-Chun Huang ${ }^{7}$, \\ Ching-Chang Fang ${ }^{8}$, Hui-Wen Lin ${ }^{2}$, Sheng-Hsiang Lin ${ }^{9,10,11}$, Chin-Feng Tsai ${ }^{1}$, \\ Chun-Hung Su${ }^{1 *}$ and Yi-Heng $\mathrm{Li}^{2 *}$ \\ ${ }^{1}$ School of Medicine, Chung Shan Medical University Hospital, Chung Shan Medical University, Taichung, Taiwan, ${ }^{2}$ College \\ of Medicine, National Cheng Kung University Hospital, National Cheng Kung University, Tainan, Taiwan, ${ }^{3}$ Kaohsiung \\ Municipal Ta-Tung Hospital and Kaohsiung Medical University Hospital, Kaohsiung, Taiwan, ${ }^{4}$ Chang Gung Memorial \\ Hospital, Chang Gung University College of Medicine, Taoyuan, Taiwan, ${ }^{5}$ Mackay Memorial Hospital, Taipei, Taiwan, \\ ${ }^{6}$ Changhua Christian Hospital, Changhua, Taiwan, ${ }^{7}$ Kaohsiung Veterans General Hospital, Fooyin University, Kaohsiung and \\ National Yang Ming University, Taipei, Taiwan, ${ }^{8}$ Tainan Municipal Hospital, Tainan, Taiwan, ${ }^{9}$ College of Medicine, Institute of \\ Clinical Medicine, National Cheng Kung University, Tainan, Taiwan, ${ }^{10}$ Department of Public Health, College of Medicine, \\ National Cheng Kung University, Tainan, Taiwan, ${ }^{11}$ Biostatistics Consulting Center, College of Medicine, National Cheng Kung \\ University Hospital, National Cheng Kung University, Tainan, Taiwan
}

Background: Dual antiplatelet therapy (DAPT) score is used to stratify ischemic and bleeding risk for antiplatelet therapy after percutaneous coronary intervention (PCl). This study assessed the association between the DAPT score and clinical outcomes in acute coronary syndrome (ACS) patients who were treated with P2Y12 inhibitor monotherapy.

Methods: A total of 498 ACS patients, with early aspirin discontinuation for various reasons and who received P2Y12 inhibitor monotherapy after PCl, were enrolled during the period from January 1, 2014 to December 31, 2018. The efficacy and safety between those with low $(<2)$ and high $(\geq 2)$ DAPT scores were compared during a 12-month follow-up after $\mathrm{PCl}$. Inverse probability of treatment weighting was used to balance the covariates between the two groups. The primary endpoint was a composite outcome of all-cause mortality, recurrent ACS or unplanned revascularization, and stroke within 12 months. The safety endpoint was major bleeding, defined as Bleeding Academic Research Consortium (BARC) 3 or 5 bleeding.

Results: The primary composite endpoint occurred in 11.56 and $14.38 \%$ of the low and high DAPT score groups, respectively. Although there was no significant difference in the primary composite endpoint between the two groups in the multivariate Cox proportional hazards models, the risk of recurrent ACS or unplanned revascularization was significantly higher in the high DAPT score group (adjusted hazard ratio [HR]: 1.900, 
95\% confidence interval [Cl]: 1.095-3.295). The safety outcome for BARC 3 or 5 bleeding was similar between the two groups.

Conclusions: Our results indicate that ACS patients receiving P2Y12 monotherapy with high DAPT score had an increased risk of recurrent ACS or unplanned revascularization.

Keywords: P2Y12 inhibitor, acute coronary syndrome, DAPT score, P2Y12 inhibitor monotherapy, clinical outcome

\section{INTRODUCTION}

Dual antiplatelet therapy (DAPT) with aspirin and P2Y12 inhibitor is the foundation therapy for acute coronary syndrome (ACS). Current guidelines recommend 12-month DAPT for patients with ACS who have received percutaneous coronary intervention (PCI) (1-3). However, DAPT-associated bleeding has raised concerns because bleeding complications increase the risk of morbidity and mortality (4-6). As patient-tailored antiplatelet therapy has become necessary, the DAPT score was developed to help physicians select patients who would benefit the most from longer or shorter DAPT after PCI (7). The DAPT scoring system includes eight positive predictors (smoking, diabetes, myocardial infarction [MI] at presentation, prior PCI or MI, paclitaxel-eluting stent, stent diameter $<3 \mathrm{~mm}$, congestive heart failure or left ventricular ejection fraction $<30 \%$, and vein graft stent) and one negative predictor (age) (7). For patients with a high DAPT score $(\geq 2)$, who had an increased ischemic risk, treatment with extended DAPT beyond 1 year resulted in a reduction in ischemic events but without an increase in bleeding (7). The DAPT score has been validated as useful for stratifying ischemia and bleeding risk in other patient groups, including Asian patients, outside the derivation cohort of the DAPT trial (8-10). Although there were some controversial results regarding its discriminating ability in one study (11), the DAPT score seems to be a clinically useful tool for determining DAPT duration after PCI.

Recently, several randomized trials evaluated the efficacy and safety of very short duration DAPT (1-3 months) followed by P2Y12 inhibitor monotherapy in patients who underwent PCI for stable coronary artery disease (CAD) and/or ACS (12-16). The rationale for using a very short period of aspirin therapy is that the benefits of intensive antiplatelet therapy with DAPT generally outweigh the risk of bleeding in the first few weeks after ACS or PCI, when the thrombogenic potential is still high. However, this benefit dissipates over time after that period and the antiplatelet potency could be enough with P2Y12 inhibitor monotherapy during later periods $(6,17)$. Overall, these clinical trials demonstrated a significant reduction in bleeding with P2Y12 inhibitor monotherapy vs. standard 12-month DAPT but no significant difference in terms of major adverse cardiovascular events (MACEs) (18). Among these clinical trials, TICO study was the first performed in Asia, which compared ticagrelor monotherapy after 3 months of DAPT vs. standard 12-month DAPT in ACS patients undergoing PCI (16). The risk of major bleeding was decreased in the ticagrelor monotherapy group but the rate of MACEs was similar to standard DAPT. Since Asian patients carry a higher bleeding risk with DAPT (19), very short
DAPT followed by P2Y12 inhibitor monotherapy may be an alternative choice for Asian ACS patients.

In the TICO study, there was a significant interaction between P2Y12 monotherapy vs. standard DAPT and the presence of multivessel disease for the primary outcome (16). In the posthoc analysis of patients with ST elevation MI in the TICO trial, the incidence of MACEs was slightly higher in the ticagrelor monotherapy group compared with 12-month DAPT in those who underwent complex PCI (4.9 vs. 2.7\%) (17). Although $\mathrm{P} 2 \mathrm{Y} 12$ inhibitor monotherapy was recommended as an optional antiplatelet strategy with standard DAPT in the 2020 European Society of Cardiology Guidelines for the management of ACS (1), there is no useful clinical outcome-predictive tool for choosing between different strategies. The efficacy of P2Y12 inhibitor monotherapy in high risk ischemic patients is also unknown. The aim of this study was to evaluate the association between DAPT score and clinical outcomes in a cohort of ACS patients undergoing PCI and who received P2Y12 inhibitor monotherapy.

\section{METHODS}

\section{Study Population}

This was a multicenter, retrospective, registration study and the detailed study design was published previously (20). In brief, we retrospectively recruited ACS patients who were admitted to the 8 major teaching hospitals in Taiwan from January 2014 to December 2018. Patients were eligible if they were $\geq 18$ years old, were admitted with a major diagnosis of ACS, including ST elevation MI, non-ST elevation MI or unstable angina, received PCI with a bare metal stent (BMS) and/or contemporary drugeluting stent (DES) implantation during hospitalization, survived to discharge, regularly followed up in an outpatient clinic for at least 1 year after discharge, and aspirin discontinuation within 6 months. We only studied a subset of ACS patients in whom aspirin was discontinued prematurely. In all enrolled patients, aspirin was prematurely discontinued within 6 months after PCI at the physician's discretion for different reasons. $\mathrm{P} 2 \mathrm{Y} 12$ inhibitor monotherapy was used thereafter in all patients with either clopidogrel $75 \mathrm{mg}$ daily or ticagrelor $90 \mathrm{mg}$ twice daily. Prasugrel was not available during the study period in Taiwan. The exclusion criteria were patients with (1) a lifethreatening malignancy with a life expectancy of $<1$ year, (2) hematological disease with bleeding tendency, (3) treatment with immunosuppressive agents, and (4) concomitant use of oral anticoagulation therapy.

All the clinical data, including coronary risk factors, major disease history, PCI procedures, and medications were collected 
from the patients' electrical medical records according to a predetermined study protocol. The timing of aspirin discontinuation was obtained from the records of medications and the aspirin treatment duration was calculated accordingly. If possible, the reasons for aspirin discontinuation were also collected from the electronic medical records. For all included patients, the DAPT scores were calculated as previously reported (7). The DAPT score was calculated by assigning points according to the patients' characteristics, including age ( 0 for age $<65$ years, -1 for age $65-$ 74 years, and -2 for age $\geq 75$ years), smoking habit ( 1 for yes and 0 for no), diabetes mellitus ( 1 for yes and 0 for no), MI at presentation ( 1 for yes and 0 for no), prior PCI or MI ( 1 for yes and 0 for no), paclitaxel-eluting stent ( 1 for yes and 0 for no), stent diameter $<3 \mathrm{~mm}$ ( 1 for yes and 0 for no), congestive heart failure or left ventricular ejection fraction $<30 \%$ ( 2 for yes and 0 for no), and vein graft stent ( 2 for yes and 0 for no) (7). All the enrolled patients were divided into 2 groups according to their DAPT score: low $(<2)$ or high $(\geq 2)$ DAPT score. A high DAPT score $(\geq 2)$ indicated that patients are at high ischemic risk and the ischemic benefits of prolonged DAPT therapy outweigh the bleeding risks. This study was conducted according to the principles expressed in the Declaration of Helsinki and was approved by the Institutional Medical Ethics Committee of National Cheng Kung University Hospital (IRB: A-ER-107-375). The principal investigators in all participating hospitals followed the study protocol strictly and the patients who did not meet the inclusion criteria were not included in this study.

\section{Follow-Up}

The follow-up information was mainly obtained from the electronic medical records of the participating hospitals. The two major clinical outcomes of ischemic and bleeding endpoints were defined. The ischemic outcome is a composite endpoint of allcause mortality, recurrent ACS or unplanned revascularization, and stroke within 12 months after the index PCI. All components of the composite endpoint were separately defined as secondary endpoints. All patients were followed up for at least 12 months after discharge or until one of the composite endpoints occurred. All these endpoint ischemic events were documented in the medical records of the patients and reported by the physicians who were responsible for patient follow-up. Recurrent ACS was defined as readmission to a hospital for management of new onset ST elevation MI, non-ST elevation MI, or unstable angina. Unplanned revascularization was defined as the first unexpected revascularization after discharge, including re-do PCI or a coronary artery bypass graft (CABG) after the index PCI due to new onset ischemic symptoms. Stroke, including ischemic or hemorrhagic stroke, was diagnosed by the occurrence of new-onset neurological symptoms and signs from neuroimaging studies. The bleeding outcome was defined as the occurrence of major bleeding as specified by the Bleeding Academic Research Consortium (BARC) type 3 and 5 bleedings (21).

\section{Statistical Analysis}

Continuous variables were expressed as mean \pm standard deviation and categorical variables were expressed as numbers and percentages. We used an unpaired Student's $t$-test for continuous variables and a chi-squared test for categorical variables to make comparisons between groups. The inverse probability of treatment weights (IPTW) method based on propensity scores was used to adjust for the imbalances in clinical characteristics between the groups, while preserving the sample size $(22,23)$. The propensity score was calculated according to the probability conditional at baseline characteristics, including age, sex, ST elevation MI status, diabetes mellitus, hypertension, hyperlipidemia, smoker, previous MI, previous PCI, previous CABG, previous ischemic stroke, previous hemorrhagic stroke, chronic kidney disease without dialysis, end stage renal disease with dialysis, heart failure, atrial fibrillation, peripheral artery disease, left ventricular ejection fraction, coronary angiography (CAG) finding, PCI procedure, location of treated lesion, stent, and medications.

In the IPTW model, we used the propensity score to generate patient-specific stabilized weights and to control for covariate imbalances. The propensity-score weight was calculated as the inverse of the propensity score for each patient. Comparisons of the clinical characteristics, CAG findings and PCI procedures, and medications between the groups were evaluated via absolute standardized mean difference (ASMD), which was calculated as the mean or proportion of a variable divided by the pooled estimate of the standard deviation of that variable. An ASMD $>0.1$ indicated a significant difference between the two groups. Cox proportional-hazard models were then adjusted for differences in the treatment groups using IPTWs derived from the propensity score, which was designated as the IPTW model. In the IPTW model after matching, the clinical characteristics with an ASMD $>0.1$ were put into the multivariate Cox proportional-hazards model for further adjustment. Because we already divided the groups by low and high DAPT scores, the criteria for the DAPT score were not in the multivariate model. Adjusted variables included body mass index $>30$, previous ischemic stroke, end-stage renal disease with dialysis, atrial fibrillation, CAG finding, PCI procedure, location of treated lesion, BMS, and statin use. Adjusted hazard ratios (HRs) and 95\% CIs were calculated. We used the same Cox proportional hazards model to estimate the $\mathrm{p}$ values for interactions in the subgroup analysis. The SAS statistical package (version 9.4 for Windows; SAS Institute, Cary, NC, USA) was used for all analyses.

\section{RESULTS}

A total of 498 ACS patients (mean age $70.18 \pm 12.84$ years, men: $71.3 \%$ ), who received PCI with stent implantation during hospitalization and survived to discharge, were included during the study period. There were 199 patients $(40 \%)$ with low $(<2)$ DAPT scores and 299 patients $(60 \%)$ with high $(\geq 2)$ DAPT scores. The mean time for aspirin treatment duration was similar between the low and high DAPT score groups (37.76 \pm 52.67 vs. $41.90 \pm 57.54$ days, $\mathrm{p}=0.471$ ). Table 1 illustrates the reasons for premature discontinuation of aspirin. The most common reason for stopping aspirin was gastrointestinal bleeding (46.59\%) with a similar percentage in both groups. Aspirin allergy and 
TABLE 1 | Reasons for premature discontinuation of aspirin.

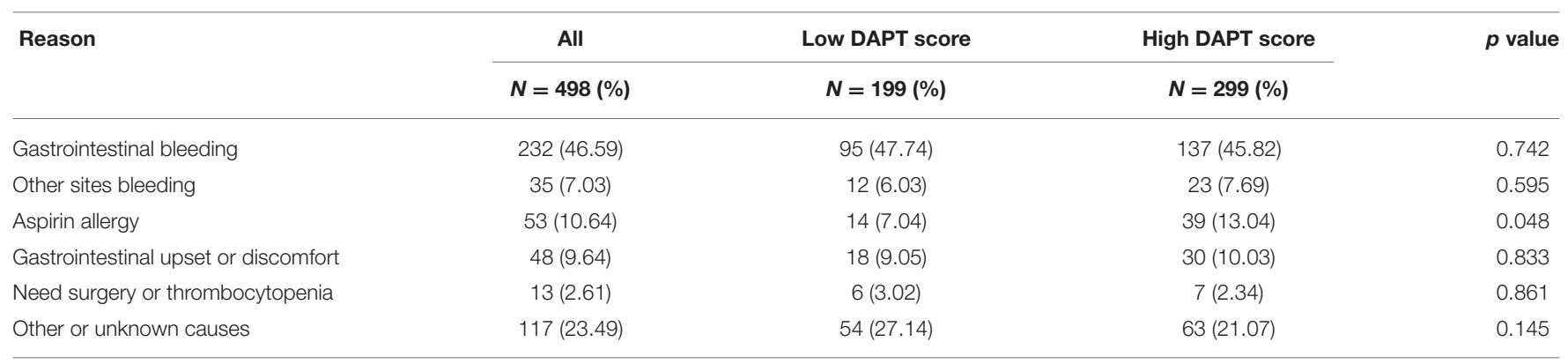

DAPT, dual antiplatelet therapy.

gastrointestinal upset were also common reasons for stopping aspirin. Aspirin allergy was significantly more common in the group with high DAPT score $(p=0.048)$, while gastrointestinal upset and discomfort were similar in both groups. Old age, anemia, or chronic use of oral non-steroidal anti-inflammatory drugs or steroids were other reasons for stopping aspirin. Unfortunately, the definite reason for stopping aspirin could not be identified in some patients from their medical records as this was a retrospective study.

Table 2 shows a comparison of baseline characteristics between the low and high DAPT score groups before and after matching. Since age, smoking, diabetes, prior PCI, prior $\mathrm{MI}$, left ventricular ejection fraction $<30 \%$, and stent diameter $<3 \mathrm{~mm}$ are the criteria included in the DAPT scoring system, it is natural to see a younger age and a higher proportion of these clinical characteristics in the high DAPT score group even after matching. The following characteristics, including body mass index, previous ischemic stroke, end stage renal disease with dialysis, atrial fibrillation, CAG finding, PCI procedure, location of treated lesion, use of stent, and use of statins, were also different between the groups and were further adjusted in the multivariate Cox proportional-hazards model for outcome evaluation.

All patients were followed up by the physicians who enrolled the patients. The mean duration of follow-up was $341.68 \pm$ 67.58 and $330.97 \pm 86.62$ days in the low and high DAPT score groups, respectively ( $p=0.123$ ). Table 3 shows the clinical outcomes during the 12-month follow-up after the index PCI. The composite ischemic outcome occurred in $11.56 \%$ of the low and $14.38 \%$ of the high DAPT score group and there was no significant difference between the groups after multivariate adjustment (adjusted HR: 1.169, 95\% CI: 0.832-1.643). For the secondary endpoint, there was also no significant difference in stroke and all-cause death between the two groups. However, the risk of recurrent ACS or unplanned revascularization was significantly higher in the high DAPT score group (adjusted HR: 1.900, 95\% CI: 1.095-3.295) compared with the low DAPT score group. BARC 3 and 5 bleeding occurred in $3.02 \%$ of the low and $4.01 \%$ of the high DAPT score group. There was no significant difference in BARC 3 and 5 bleeding (adjusted HR: 1.206, 95\% CI: 0.623-2.335) between the two groups.

Figure 1 shows the subgroup analysis results regarding sex, clopidogrel or ticagrelor, hypertension, chronic kidney disease, 3-vessel disease, single or multiple-lesion intervention, and DES between the two groups. The criteria in the DAPT score, such as age, smoking, diabetes, prior PCI, prior MI, left ventricular ejection fraction $<30 \%$, and stent diameter $<3 \mathrm{~mm}$, were not used for the subgroup analysis. In the subgroup analysis, patients with high DAPT score had a higher risk of primary composite endpoint in the subgroups of chronic kidney disease, 3-vessel disease, and DES ( $p$ for interaction $<0.05$ ). There was a borderline interaction between those treated with ticagrelor or clopidogrel $(p$ for interaction $=0.052$ ).

\section{DISCUSSION}

In this study, we assessed the association between the DAPT score and the clinical outcomes in ACS patients receiving P2Y12 inhibitor monotherapy after index PCI. We found that the DAPT score was useful for determining the ischemic risk in these patients. Although previous clinical trials found that the efficacy of P2Y12 inhibitor monotherapy for preventing ischemic events was comparable to standard 12-month DAPT, the present study demonstrated that patients with high DAPT score still had a significantly higher rate of recurrent ACS or unplanned revascularization compared with those with low DAPT score.

After its development in the DAPT trial, the DAPT score has been extensively investigated with regard to its ability to stratify ischemic risk in a wide variety of patient groups who have received PCI (8-11). Most of the studies confirmed that patients with a high DAPT score have a higher incidence of ischemic events when compared with patients with a low DAPT score. For the strategy of very short DAPT (1-3 months) followed by P2Y12 inhibitor monotherapy, the influence of the DAPT score remains unclear. GLOBAL LEADERS trial is a randomized study comparing 1 year of DAPT therapy (aspirin plus clopidogrel or ticagrelor) followed by 1 year of aspirin monotherapy with 1 month of DAPT therapy (aspirin plus ticagrelor) followed by 23 months of ticagrelor, among patients undergoing PCI for stable CAD or ACS (12). A recent study analyzed the clinical outcomes from the second year follow up with aspirin or ticagrelor monotherapy in the GLOBAL LEADERS trial. It demonstrated that patients with high DAPT score had a significantly higher rate of the composites of MI or stent thrombosis ( $0.70 \%$ vs. $1.55 \%$, $p<0.0001)$ and a similar rate of BARC type 3 or 5 bleeding (24). 
TABLE 2 | Baseline characteristics of patients with different DAPT scores.

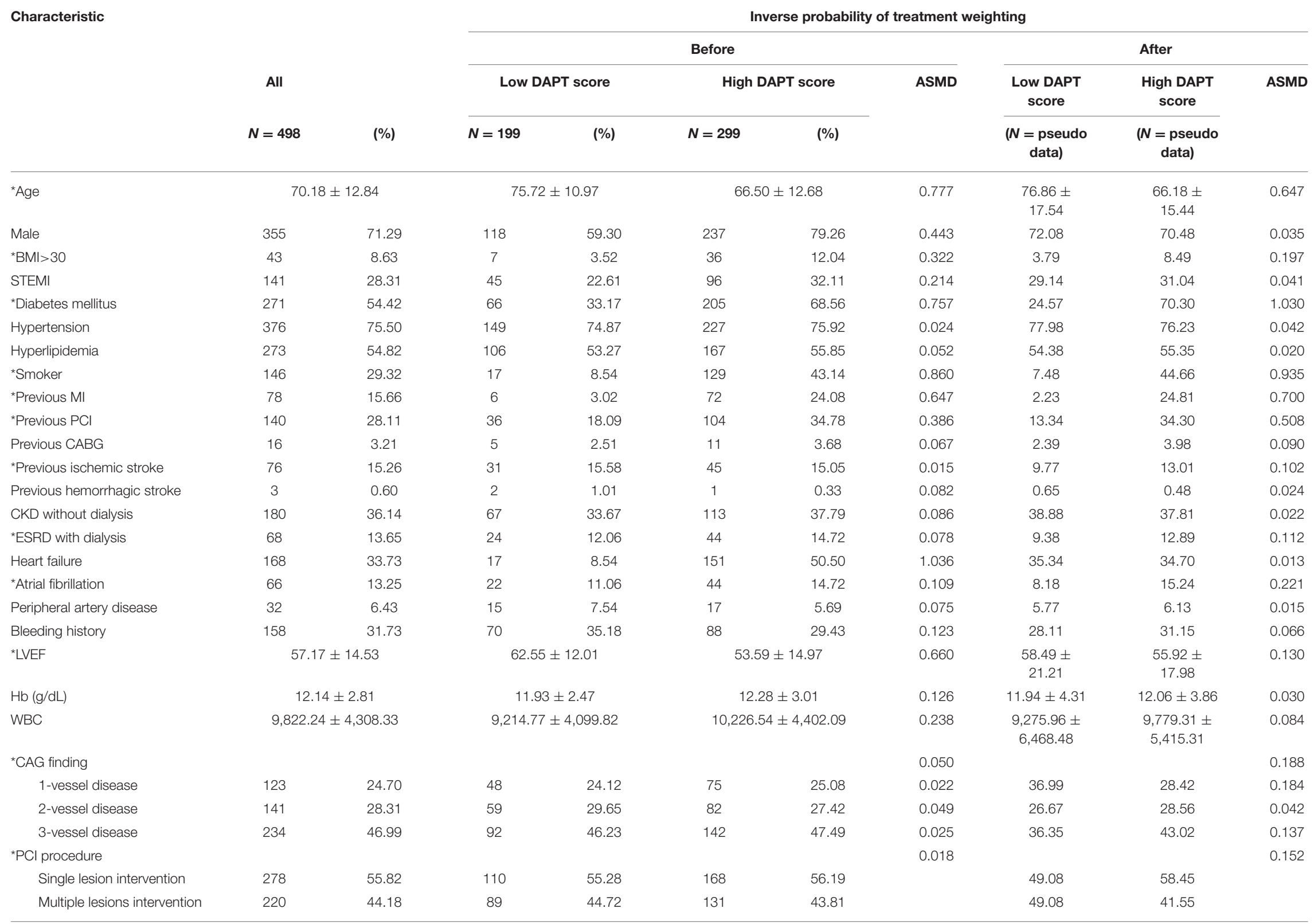




\begin{tabular}{|c|c|c|c|c|c|c|c|c|c|c|}
\hline \multirow[t]{4}{*}{ Characteristic } & & \multirow[b]{4}{*}{$(\%)$} & \multicolumn{8}{|c|}{ Inverse probability of treatment weighting } \\
\hline & \multirow{3}{*}{$\begin{array}{c}\text { All } \\
N=498\end{array}$} & & \multicolumn{5}{|c|}{ Before } & \multicolumn{3}{|c|}{ After } \\
\hline & & & \multicolumn{2}{|c|}{ Low DAPT score } & \multicolumn{2}{|c|}{ High DAPT score } & \multirow[t]{2}{*}{ ASMD } & \multirow{2}{*}{$\begin{array}{c}\begin{array}{c}\text { Low DAPT } \\
\text { score }\end{array} \\
\begin{array}{c}(\mathrm{N}=\text { pseudo } \\
\text { data })\end{array}\end{array}$} & \multirow{2}{*}{$\begin{array}{c}\begin{array}{c}\text { High DAPT } \\
\text { score }\end{array} \\
\begin{array}{c}(\mathrm{N}=\text { pseudo } \\
\text { data })\end{array}\end{array}$} & \multirow[t]{2}{*}{ ASMD } \\
\hline & & & $N=199$ & $(\%)$ & $N=299$ & (\%) & & & & \\
\hline \multicolumn{11}{|l|}{ *Location of lesion treated } \\
\hline LM & 38 & 7.63 & 17 & 8.54 & 21 & 7.02 & 0.057 & 4.83 & 6.53 & 0.073 \\
\hline LAD & 319 & 64.06 & 130 & 65.33 & 189 & 63.21 & 0.044 & 55.26 & 63.18 & 0.162 \\
\hline LCX & 194 & 38.96 & 78 & 39.20 & 116 & 38.80 & 0.008 & 31.83 & 38.42 & 0.138 \\
\hline $\mathrm{RCA}$ & 234 & 46.99 & 94 & 47.24 & 140 & 46.82 & 0.008 & 52.45 & 44.55 & 0.159 \\
\hline$S V G$ & 2 & 0.40 & 0 & 0.00 & 2 & 0.67 & 0.116 & - & 0.90 & \\
\hline \multicolumn{11}{|l|}{ *Stent } \\
\hline Bare metal stent & 214 & 42.97 & 80 & 40.20 & 134 & 44.82 & 0.094 & 35.84 & 43.62 & 0.159 \\
\hline Everolimus-eluting stent & 93 & 18.67 & 35 & 17.59 & 58 & 19.40 & 0.047 & 17.16 & 19.61 & 0.063 \\
\hline Zotarolimus-eluting stent & 99 & 19.88 & 43 & 21.61 & 56 & 18.73 & 0.072 & 17.40 & 19.26 & 0.048 \\
\hline Biolimus-eluting stent & 26 & 5.22 & 14 & 7.04 & 12 & 4.01 & 0.133 & 7.07 & 4.75 & 0.098 \\
\hline Sirolimus-eluting stent & 65 & 13.05 & 31 & 15.58 & 34 & 11.37 & 0.123 & 17.55 & 12.00 & 0.157 \\
\hline Stent $<3 \mathrm{~mm}$ & 200 & 40.16 & 60 & 30.15 & 140 & 46.82 & 0.348 & 20.12 & 52.30 & 0.711 \\
\hline \multicolumn{11}{|l|}{ Medications } \\
\hline Clopidogrel & 271 & 54.42 & 118 & 59.30 & 153 & 51.17 & 0.164 & 51.15 & 46.26 & 0.098 \\
\hline Ticagrelor & 227 & 45.58 & 81 & 40.70 & 146 & 48.83 & 0.164 & 48.85 & 53.74 & 0.098 \\
\hline Beta blocker & 367 & 73.69 & 133 & 66.83 & 234 & 78.26 & 0.258 & 75.79 & 73.35 & 0.056 \\
\hline RAS inhibitor & 283 & 56.83 & 105 & 52.76 & 178 & 59.53 & 0.137 & 57.64 & 56.88 & 0.015 \\
\hline *Statin & 405 & 81.33 & 153 & 76.88 & 252 & 84.28 & 0.188 & 75.87 & 82.19 & 0.156 \\
\hline PPI use & 203 & 40.76 & 96 & 48.24 & 107 & 35.79 & 0.254 & 39.63 & 40.53 & 0.018 \\
\hline
\end{tabular}

BMI, body mass index; CABG, coronary artery bypass graft; CAG, coronary angiography; CKD, chronic kidney disease; DAPT, dual antiplatelet therapy; ESRD, end stage renal disease; $L A D$, left anterior descending artery, $L C X$, left circumflex artery; LM, left main artery; LVEF, left ventricular ejection fraction; MI, myocardial infarction; PCl, percutaneous coronary intervention; PPI, proton pump inhibitor; RAS, renin angiotensin system; RCA, right coronary artery; STEMI, ST-segment elevation myocardial infarction; SVG, saphenous vein graft; ASMD, absolute standardized difference.

"ASMD >0.1 between the groups. 
TABLE 3 | Clinical outcomes at 12-months follow-up.

\begin{tabular}{|c|c|c|c|c|c|c|c|}
\hline \multirow[t]{2}{*}{ Outcome } & All & Low DAPT score & High DAPT score & Crude HR & $p$ value & Adjusted HR & $p$ value \\
\hline & $N=498$ & $N=199$ (Ref) & $N=299$ & $(95 \% \mathrm{Cl})$ & \multicolumn{3}{|c|}{$(95 \% \mathrm{Cl})$} \\
\hline Primary composite endpoint & 66 (13.25) & $23(11.56)$ & $43(14.38)$ & 0.792 (0.579-1.082) & 0.143 & 1.169 (0.832-1.643) & 0.367 \\
\hline \multicolumn{8}{|l|}{ Secondary endpoint } \\
\hline $\begin{array}{l}\text { Recurrent ACS or } \\
\text { unplanned revascularization }\end{array}$ & $41(8.23)$ & $12(6.03)$ & $29(9.70)$ & $1.965(1.145-3.372)$ & 0.014 & 1.900 (1.095-3.295) & 0.022 \\
\hline Stroke & $1(0.20)$ & 0 & $1(0.33)$ & - & & - & \\
\hline All-cause death & $24(4.82)$ & $11(5.53)$ & $13(4.35)$ & $0.426(0.277-0.654)$ & $<0.001$ & $0.758(0.465-1.237)$ & 0.268 \\
\hline BARC 3 or 5 bleeding & $18(3.61)$ & $6(3.02)$ & $12(4.01)$ & $1.341(0.709-2.539)$ & 0.367 & $1.206(0.623-2.335)$ & 0.578 \\
\hline
\end{tabular}

ACS, acute coronary syndrome; BARC, Bleeding Academic Research Consortium; DAPT, dual antiplatelet therapy.

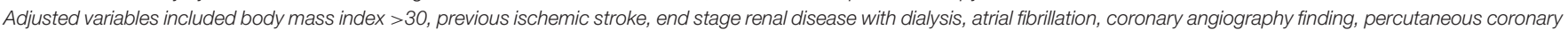
intervention procedure, location of treated lesion, bare metal stent, and statin.

\begin{tabular}{|c|c|c|c|c|}
\hline \multicolumn{4}{|c|}{ Primary composite endpoint } & \multirow{2}{*}{$\begin{array}{c}P \text { for } \\
\text { interaction }\end{array}$} \\
\hline & & & Hazard Ratio $(95 \% \mathrm{Cl})$ & \\
\hline Gender (Male) & & $\longmapsto$ & $1.628(1.062-2.495)$ & \multirow[t]{2}{*}{0.316} \\
\hline (Female) & $\vdash$ & $\longrightarrow$ & $0.641(0.315-1.304)$ & \\
\hline Clopidogrel & $\longmapsto$ & $\longrightarrow$ & $1.170(0.741-1.847)$ & \multirow[t]{2}{*}{0.052} \\
\hline Ticagrelor & & $\longmapsto$ & $2.045(1.032-4.051)$ & \\
\hline Hypertension & & & & \multirow[t]{3}{*}{0.264} \\
\hline Yes & 4 & $\longrightarrow$ & $0.984(0.672-1.440)$ & \\
\hline No & & $\longrightarrow$ & $1.661(0.673-4.100)$ & \\
\hline CKD & & & & \multirow{3}{*}{0.011} \\
\hline Yes & $\longmapsto$ & + & $1.157(0.748-1.790)$ & \\
\hline No & $\longmapsto$ & $\longrightarrow$ & $1.322(0.694-2.518)$ & \\
\hline CAG finding & & & & \multirow[t]{3}{*}{$<0.001$} \\
\hline$<3$-vessel disease & $\longmapsto$ & & $0.638(0.395-1.032)$ & \\
\hline 3-vessel disease & & $\longmapsto$ & $2.562(1.413-4.646)$ & \\
\hline $\mathrm{PCl}$ procedure & & & & \multirow[t]{3}{*}{0.249} \\
\hline Single-lesion intervention & & -1 & $0.645(0.381-1.092)$ & \\
\hline Multi-lesion intervention & & $\longmapsto$ & $2.938(1.710-5.046)$ & \\
\hline DES & & & & \multirow[t]{4}{*}{$<0.001$} \\
\hline Yes & & $\longmapsto$ & $2.252(1.175-4.319)$ & \\
\hline \multirow{3}{*}{0.2} & $\longmapsto$ & $\longrightarrow$ & $0.969(0.611-1.538)$ & \\
\hline & $\begin{array}{ll}1 & 1 \\
0.5 & 1\end{array}$ & 2 & & \\
\hline & $\begin{array}{l}\text { High DAPT score } \\
\text { better }\end{array}$ & $\begin{array}{c}\text { Low DAPT score } \\
\text { better }\end{array}$ & & \\
\hline
\end{tabular}

The authors concluded that the DAPT score can stratify ischemic risk but not bleeding risk in a contemporary PCI population during the second year.

Our study only included ACS patients receiving P2Y12 inhibitor monotherapy (ticagrelor or clopidogrel), and we observed the first year outcomes after PCI. We initially hypothesized that a high DAPT score would predict higher composite ischemic events, but the results were not as expected. The potential reasons for this are the limited follow-up time (12 months) and the small case number. However, we did find that 
the risk of recurrent ACS or unplanned revascularization was significantly higher in the high DAPT score group. In addition, our study also found that the DAPT score could not stratify bleeding risk in ACS patients undergoing PCI, which was similar to the results of the GLOBAL LEADERS trial (24). Subgroup analysis revealed that there were more ischemic events in the high DAPT score group with 3-vessel disease, and coronary anatomy complexity is a known risk factor for MACEs after PCI $(25,26)$.

Our study results indicate that early discontinuation of aspirin with P2Y12 inhibitor monotherapy should be the last resort for ACS patients with 3-vessel disease and a high DAPT score because the high ischemic risk is still a major concern. Overall, the patients receiving BMS deployment had higher ischemic events than those receiving DES deployment (recurrent ACS or unplanned revascularization: BMS 11.21 vs. DES 6.72\%). However, in the subgroup analysis, we found that patients receiving P2Y12 inhibitor monotherapy with ticagrelor and DES deployment, had higher primary composite endpoints in the high DAPT score group. Probable reasons to explain this result are that the choice of P2Y12 inhibitor and stent in real-world practice are based on the clinician's experience and the local insurance system. Ticagrelor is more expensive than clopidogrel. A previous real-world observation study of ACS in Taiwan demonstrated that ticagrelor offers a better protective effect against ischemic events when compared with clopidogrel (27). However, BMS is still commonly used in Taiwan because the Taiwan National Health Insurance only reimburses the price of BMS. Patients using DES have to pay the price difference, which is around US $\$ 1,500$ to US $\$ 2,000$ for one DES (28). Therefore, it is likely that physicians in Taiwan are prone to choosing ticagrelor and DES for ACS patients with a higher ischemic risk and recurrent events.

To the best of our knowledge, this is the first study to assess the ability of the DAPT score to stratify ischemic and bleeding risk in ACS patients with P2Y12 inhibitor monotherapy undergoing PCI in Asia. However, our study did have several limitations. First, our study was a retrospective, nonrandomized, observational study. The unadjusted confounding factors were unavoidable, even though a propensity score-matched analysis was used to compensate for this. Second, the case number was relatively small in our study, which may have caused selection bias of the included patients. Standard 12-month DAPT is still the recommended therapy for ACS patients who undergo PCI in Taiwan (29). Therefore, it is difficult to collect large case numbers of patients with only P2Y12 inhibitor monotherapy from realworld practice. Third, there was only a 12-month follow-up after PCI. A longer follow-up duration may be necessary to determine the definite association of the DAPT score with the clinical outcomes. Fourth, although the guidelines recommend new-generation P2Y12 inhibitors and DES for ACS patients undergoing PCI, BMS and clopidogrel are still commonly used in Taiwan for various reasons. Our study results may be different if all patients were treated with ticagrelor and DES. Fifth, we did not know the exact cause of death in all 24 patients with mortality. Some patients died from sepsis/pneumonia, cancer, and respiratory failure, but not cardiovascular causes. Finally, our results may not be applicable to non-Asian patients.

In conclusion, ACS patients receiving early P2Y12 monotherapy with high DAPT score had a higher risk of recurrent ACS or unplanned revascularization compared with those with low DAPT score. The risk of major bleeding was similar between those with low and high DAPT scores. This study suggests that the DAPT score is validated for predicting cardiovascular events in ACS patients undergoing PCI with short DAPT treatment followed by P2Y12 inhibitor monotherapy.

\section{DATA AVAILABILITY STATEMENT}

The original contributions presented in the study are included in the article/supplementary material, further inquiries can be directed to the corresponding authors.

\section{ETHICS STATEMENT}

The studies involving human participants were reviewed and approved by the Institutional Medical Ethics Committee of National Cheng Kung University Hospital (IRB: A-ER107-375). Written informed consent was not required for this study, in accordance with the local legislation and institutional requirements.

\section{AUTHOR CONTRIBUTIONS}

M-YH, W-HF, C-FT, C-HS, S-WH, C-WC, H-IY, C-PC, $\mathrm{W}-\mathrm{CH}, \mathrm{C}-\mathrm{CF}, \mathrm{I}-\mathrm{CH}$, and $\mathrm{Y}-\mathrm{HL}$ were responsible for the conceptualization. Data curation was done by $\mathrm{M}-\mathrm{YH}, \mathrm{P}-\mathrm{WC}$, W-HF, C-FT, C-HS, S-WH, C-WC, H-IY, C-PC, W-CH, C-CF, I-CH, and Y-HL. M-YH, P-WC, W-HF, C-HS, S-WH, C-WC, $\mathrm{H}-\mathrm{IY}, \mathrm{C}-\mathrm{PC}, \mathrm{W}-\mathrm{CH}, \mathrm{C}-\mathrm{CF}, \mathrm{I}-\mathrm{CH}$, and Y-HL performed the formal analysis. Funding acquisition was taken care of by $\mathrm{W}-\mathrm{HF}$ and I-CH. M-YH, P-WC, W-HF, C-HS, H-WL, and Y-HL carried out the investigation. P-WC, W-HF, H-WL, and S-HL took care of the methodology. Project administration was the responsibility of C-HS, P-WC, W-HF, and Y-HL. H-WL and S-HL handled the software. C-HS, C-FT, S-HL, I-CH, and Y-HL supervised the study. C-FT, H-WL, S-HL, and I-CH performed the validation. C-FT, S-HL, and I-CH visualized the study. Writing of the original draft was done by S-WH, P-WC, C-HS, and Y-HL. C-HS, S-WH, Y-HL, and P-WC reviewed and edited the study. All authors contributed to the article and approved the submitted version.

\section{FUNDING}

The authors gratefully acknowledge the support of Kaohsiung Municipal Ta-Tung Hospital (kmtth-109-009). 


\section{REFERENCES}

1. Collet JP, Thiele H, Barbato E, Barthelemy O, Bauersachs J, Bhatt DL, et al. 2020 ESC Guidelines for the management of acute coronary syndromes in patients presenting without persistent ST-segment elevation. Eur Heart J. (2021) 42:1289-367. doi: 10.1093/eurheartj/ehaa575

2. Valgimigli M, Bueno H, Byrne RA, Collet JP, Costa F, Jeppsson A, et al. 2017 ESC focused update on dual antiplatelet therapy in coronary artery disease developed in collaboration with EACTS: The Task Force for dual antiplatelet therapy in coronary artery disease of the European Society of Cardiology (ESC) and of the European Association for Cardio-Thoracic Surgery (EACTS). Eur Heart J. (2018) 39:213-60. doi: 10.1093/eurheartj/ehx419

3. Levine GN, Bates ER, Bittl JA, Brindis RG, Fihn SD, Fleisher LA, et al. 2016 ACC/AHA guideline focused update on duration of dual antiplatelet therapy in patients with coronary artery disease: a report of the American College of Cardiology/American Heart Association Task Force on Clinical Practice Guidelines. J Am Coll Cardiol. (2016) 68:1082115. doi: $10.1016 /$ j.jacc.2016.03.513

4. Sharma A, Hagstrom E, Wojdyla DM, Neely ML, Harrington RA, Wallentin L, et al. Clinical consequences of bleeding among individuals with a recent acute coronary syndrome: insights from the APPRAISE-2 trial. Am Heart J. (2019) 215:106-13. doi: 10.1016/j.ahj.2019.05.004

5. van der Sangen NMR, Rozemeijer R, Chan Pin Yin DRPP, Valgimigli M, Windecker S, James SK, et al. Patient-tailored antithrombotic therapy following percutaneous coronary intervention. Eur Heart J. (2021) 42:103846. doi: 10.1093/eurheartj/ehaa1097

6. Rodriguez F, Harrington RA. Management of antithrombotic therapy after acute coronary syndromes. N Engl J Med. (2021) 384:452-60. doi: 10.1056/NEJMra1607714

7. Yeh RW, Secemsky EA, Kereiakes DJ, Normand SL, Gershlick AH, Cohen DJ, et al. Development and validation of a prediction rule for benefit and harm of dual antiplatelet therapy beyond 1 year after percutaneous coronary intervention. JAMA. (2016) 315:1735-49. doi: 10.1001/jama.2016.3775

8. Piccolo R, Gargiulo G, Franzone A, Santucci A, Ariotti S, Baldo A, et al. Use of the dual-antiplatelet therapy score to guide treatment duration after percutaneous coronary intervention. Ann Intern Med. (2017) 167:1725. doi: 10.7326/M16-2389

9. Yoshikawa Y, Shiomi H, Watanabe H, Natsuaki M, Kondo H, Tamura T, et al. Validating utility of dual antiplatelet therapy score in a large pooled cohort from 3 Japanese percutaneous coronary intervention studies. Circulation. (2018) 137:551-62. doi: 10.1161/CIRCULATIONAHA.117.028924

10. Montalto C, Ferlini M, Casula M, Mandurino-Mirizzi A, Costa F, Leonardi S, et al. DAPT score to stratify ischemic and bleeding risk after percutaneous coronary intervention: an updated systematic review, meta-analysis, and meta-regression of 100,211 Patients. Thromb Haemost. (2021) 121:6879. doi: $10.1055 / \mathrm{s}-0040-1721145$

11. Ueda P, Jernberg T, James S, Alfredsson J, Erlinge D, Omerovic E, et al. External validation of the DAPT score in a nationwide population. J Am Coll Cardiol. (2018) 72:1069-78. doi: 10.1016/j.jacc.2018.06.023

12. Vranckx P, Valgimigli M, Jüni P, Hamm C, Steg PG, Heg D, et al. Ticagrelor plus aspirin for 1 month, followed by ticagrelor monotherapy for 23 months vs aspirin plus clopidogrel or ticagrelor for 12 months, followed by aspirin monotherapy for 12 months after implantation of a drug-eluting stent: a multicentre, open-label, randomised superiority trial. Lancet. (2018) 392:9409. doi: 10.1016/S0140-6736(18)31858-0

13. Mehran R, Baber U, Sharma SK, Cohen DJ, Angiolillo DJ, Briguori C, et al. Ticagrelor with or without aspirin in high-risk patients after PCI. $N$ Engl J Med. (2019) 381:2032-42. doi: 10.1056/NEJMoa1908419

14. Watanabe H, Domei T, Morimoto T, Natsuaki M, Shiomi H, Toyota T, et al. Effect of 1-month dual antiplatelet therapy followed by clopidogrel vs 12month dual antiplatelet therapy on cardiovascular and bleeding events in patients receiving PCI: The STOPDAPT-2 randomized clinical trial. JAMA. (2019) 321:2414-27. doi: 10.1001/jama.2019.8145

15. Hahn JY, Song YB, Oh JH, Chun WJ, Park YH, Jang WJ, et al. Effect of P2Y12 inhibitor monotherapy vs dual antiplatelet therapy on cardiovascular events in patients undergoing percutaneous coronary intervention: The SMART-CHOICE randomized clinical trial. JAMA. (2019) 321:242837. doi: 10.1001/jama.2019.8146
16. Kim BK, Hong SJ, Cho YH, Yun KH, Kim YH, Suh Y, et al. Effect of ticagrelor monotherapy vs ticagrelor with aspirin on major bleeding and cardiovascular events in patients with acute coronary syndrome. JAMA. (2020) 323:240716. doi: 10.1001/jama.2020.7580

17. Feng WH, Hsieh IC Li YH. P2Y12 inhibitor monotherapy after percutaneous coronary intervention: is it safe to abandon aspirin? Acta Cardiol Sin. (2021) 37:1-8. doi: 10.6515/ACS.202101_37(1).20200806A

18. Giacoppo D, Matsuda Y, Fovino LN, D’Amico G, Gargiulo G, Byrne RA, et al. Short dual antiplatelet therapy followed by P2Y12 inhibitor monotherapy vs. prolonged dual antiplatelet therapy after percutaneous coronary intervention with second-generation drug-eluting stents: a systematic review and meta-analysis of randomized clinical trials. Eur Heart J. (2021) 42:30819. doi: 10.1093/eurheartj/ehaa739

19. Kang J, Park KW, Palmerini T, Stone GW, Lee MS, Colombo A, et al. Racial differences in ischaemia/bleeding risk trade-off during anti-platelet therapy: individual patient level landmark meta-analysis from seven RCTs. Thromb Haemost. (2019) 119:149-62. doi: 10.1055/s-0038-1676545

20. Chen PW, Feng WH, Ho MY, Su CH, Huang SW, Cheng CW, et al. P2Y12 inhibitor monotherapy with clopidogrel versus ticagrelor in patients with acute coronary syndrome undergoing percutaneous coronary intervention. $J$ Clin Med. (2020) 9:1657. doi: 10.3390/jcm9061657

21. Mehran R, Rao SV, Bhatt DL, Gibson CM, Caixeta A, Eikelboom J, et al. Standardized bleeding definitions for cardiovascular clinical trials: a consensus report from the Bleeding Academic Research Consortium. Circulation. (2011) 123:2736-47. doi: 10.1161/CIRCULATIONAHA.110.009449

22. Austin PC. An introduction to propensity score methods for reducing the effects of confounding in observational studies. Multivariate Behav Res. (2011) 46:399-424. doi: 10.1080/00273171.2011.568786

23. Burden A, Roche N, Miglio C, Hillyer EV, Postma DS, Herings RM, et al. An evaluation of exact matching and propensity score methods as applied in a comparative effectiveness study of inhaled corticosteroids in asthma. Pragmat Obs Res. (2017) 8:15-30. doi: 10.2147/POR.S122563

24. Chichareon P, Modolo R, Kawashima H, Takahashi K, Kogame N, Chang CC, et al. DAPT score and the impact of ticagrelor monotherapy during the second year after PCI. JACC Cardiovasc Interv. (2020) 13:63446. doi: $10.1016 /$ j.jcin.2019.12.018

25. Valle JA, Glorioso TJ, Bricker R, Barón AE, Armstrong EJ, Bhatt DL, et al. Association of coronary anatomical complexity with clinical outcomes after percutaneous or surgical revascularization in the veterans affairs clinical assessment reporting and tracking program. JAMA Cardiol. (2019) 4:72735. doi: 10.1001/jamacardio.2019.1923

26. Mohamed MO, Polad J, Hildick-Smith D, Bizeau O, Baisebenov RK, Roffi $\mathrm{M}$, et al. Impact of coronary lesion complexity in percutaneous coronary intervention: one-year outcomes from the large, multicentre e-Ultimaster registry. EuroIntervention. (2020) 16:603-12. doi: 10.4244/EIJ-D-20-00361

27. Lee $\mathrm{CH}$, Cheng $\mathrm{CL}$, Kao Yang $\mathrm{YH}$, Chao TH, Chen JY Li YH. Cardiovascular and bleeding risks in acute myocardial infarction newly treated with ticagrelor vs. clopidogrel in Taiwan Circ J. (2018) 82:74756. doi: $10.1253 /$ circj.CJ-17-0632

28. Wu CK, Juang JJ, Chiang JY Li YH, Tsai CT, Chiang FT. The Taiwan Heart Registries: its influence on cardiovascular patient care. J Am Coll Cardiol. (2018) 71:1273-83. doi: 10.1016/j.jacc.2018.02.006

29. Li YH, Lee CH, Huang WC, Wang YC, Su CH, Sung PH, et al. 2020 focused update of the 2012 Guidelines of the Taiwan Society of Cardiology for the management of ST-segment elevation myocardial infarction. Acta Cardiol Sin. (2020) 36:285-307. doi: 10.6515/ACS.202007_36(4).20200619A

Conflict of Interest: The authors declare that the research was conducted in the absence of any commercial or financial relationships that could be construed as a potential conflict of interest.

Publisher's Note: All claims expressed in this article are solely those of the authors and do not necessarily represent those of their affiliated organizations, or those of the publisher, the editors and the reviewers. Any product that may be evaluated in this article, or claim that may be made by its manufacturer, is not guaranteed or endorsed by the publisher. 
Copyright (C) 2022 Huang, Chen, Feng, Hsieh, Ho, Cheng, Yeh, Chen, Huang, Fang, Lin, Lin, Tsai, Su and Li. This is an open-access article distributed under the terms of the Creative Commons Attribution License (CC BY). The use, distribution or reproduction in other forums is permitted, provided the original author(s) and the copyright owner(s) are credited and that the original publication in this journal is cited, in accordance with accepted academic practice. No use, distribution or reproduction is permitted which does not comply with these terms. 